\title{
Prevalence and Risk Factors for Lumbar Spondylosis and Its Association with Low Back Pain among Rural Korean Residents
}

\author{
Sung Yeon Lee, M.D., ${ }^{1, *}$ Nam H. Cho, M.D., Ph.D., ${ }^{2,}$ Young Ok Jung, M.D., Ph.D., ${ }^{3}$ Young Il Seo, M.D., Ph.D., ${ }^{1,+}$ \\ Hyun Ah Kim, M.D., Ph.D.' \\ Division of Rheumatology, Department of Internal Medicine, Hallym University Sacred Heart Hospital, Anyang, Korea \\ Department of Preventive Medicine, ${ }^{2}$ Ajou University School of Medicine, Suwon, Korea \\ Division of Rheumatology, ${ }^{3}$ Department of Internal Medicine, Kangnam Sacred Heart Hospital, Seoul, Korea
}

Objective : The purpose of this study was to investigate the prevalence of and the relevant risk factors for lumbar spondylosis (LS) among middle-aged and elderly rural Korean residents and to explore the association between radiographic LS and lower back pain (LBP) in relation to age and gender.

Methods : This community-based, cross-sectional study evaluated 1512 subjects with available radiograph. The prevalence of LBP was obtained using a questionnaire and disability resulting from LBP was measured using a validated Korean version of the Oswestry disability index (ODI). In lumbar spine radiographs, vertebral levels from $L 1 / 2$ to $L 4 / 5$ were evaluated for the presence of osteophytes and joint-space narrowing (JSN), and Kellgren-Lawrence (KL) grading was applied.

Results : Of 4261 subjects aged 40-79 years, data from 1512 subjects were included. The prevalence of radiographic LS indicated by grade $\geq 2$ osteophytes and JSN were 53.9 and $15.8 \%$, respectively. Seventy-three percent of subjects had KL grade $\geq 2$ spondylosis and LBP was present in $36.5 \%$ of subjects. Although LS was more common among males, the prevalence of LBP was higher among females. Age, male gender and history of hand or knee arthritis were risk factors for LS. LS was significantly associated with LBP mostly among females over 60 years old and correlated with the ODI after adjusting for age and gender.

Conclusion : Our study among rural Korean residents revealed a high prevalence of LS and LBP. The association between LS and LBP was observed mostly among females and LS was significantly correlated with the severity of back pain.

Key Words : Prevalence · Risk factors · Lumbar spondylosis · Lower back pain.

- Received: May 24, 2016 • Accepted: October 1, 2016

- Address for reprints :

Young II Seo, M.D., Ph.D.

Division of Rheumatology, Department of Internal Medicine, Hallym University Sacred Heart Hospital, 22 Gwanpyeong-ro 170beon-gil, Dongan-gu, Anyang 14068, Korea Tel : 82-31-380-3713, Fax : 82-31-386-2269, E-mail : yiseo@hallym.or.kr

Hyun Ah Kim, M.D., Ph.D.

Tel : 82-31-380-1826, Fax : 82-31-381-8812, E-mail : kimha@hallym.ac.kr

\footnotetext{
* Sung Yeon Lee and Nam H. Cho is co-first author.

${ }^{\dagger}$ Young II Seo and Hyun Ah Kim is co-corresponding author.
}

This is an Open Access article distributed under the terms of the Creative Commons Attribution Non-Commercial License (http://creativecommons.org/licenses/by-nc/4.0) which permits unrestricted non-commercial use, distribution, and reproduction in any medium, provided the original work is properly cited. 


\section{INTRODUCTION}

Lower back pain (LBP), an important public health problem in industrialized societies, is associated with substantial healthcare and societal costs. Lumbar spondylosis (LS) is common with aging and is considered to be related to LBP and disability. LS is characterized radiologically by the presence of osteophytes, endplate sclerosis, and disc-space narrowing ${ }^{9)}$. A previous systematic review showed that LS could be a risk factor for LBP in adults, with odds ratios (ORs) varying from 1.3 to $3.2^{12)}$. The prevalence of LS in Asia has been scarcely reported, although both population aging and economic growth have occurred at a much higher rate in Asian countries such as South Korea. In addition, data on risk factors of LS in Asian populations are scarce. Yoshimura et al. demonstrated that the prevalence of LS is higher in the UK than in Japan ${ }^{15)}$. Differences in the prevalence of osteophytosis as well as disc degeneration were noted, suggesting ethnic differences in spinal joint involvement. A recent Japanese study involving 3040 elderly participants reported the prevalence of LS, defined as at least one intervertebral level graded as Kellgren-Lawrence (KL) grade $\geq 2$, as $80.6 \%$ among males and $64.6 \%$ among females ${ }^{16)}$. The prevalence increased with age, and was significantly higher among males than among females in all areas surveyed (urban, mountainous, and seacoast).

In this cross-sectional, observational study, we sought to determine the prevalence of LS among middle-aged and elderly rural Korean residents. We also examined the relevant risk factors for LS. Finally, the association between the different individual radiographic features of LS, including osteophytes and disc-space narrowing, and LBP in relation to age and gender was explored.

\section{MATERIALS AND METHODS}

\section{Study population}

As part of the ongoing prospective Korean Health and Genome Study, a rural farming community (Anseong) in South
Korea was selected in year 2001. The eligibility criteria included age 40-79 years, residence within the borders of the survey area for at least 6 months before testing, and mental and physical ability to participate. Cluster sampling using a bracket survey method was conducted among 132906 residents using a random sampling method and the local telephone directory. Participants were followed every three year. This study included 4261 subjects surveyed during the third wave (year 2006-2008). After excluding 80 subjects who were ineligible or who refused to participate in the LBP study, data for LBP were available for 4181 subjects. Among these, 2000 subjects were randomly selected for spinal radiography. Although selected subjects were significantly older compared to non-selected subjects (59.0 years vs. 54.2 years), other clinical variables including the prevalence of LBP were similar between the 2 groups (Supplementary Table 1). After excluding 488 subjects who could not be evaluated due to clerical error or poor film quality (190, poor film quality due to inadequate penetration or poor positioning of the subject, 38 , missing low back pain information, 260, missing Oswestry back pain index data), data for 1512 subjects were available. This study included only subjects with complete data set, because we deemed it not appropriate to use imputation since we were not sure whether the missing occurred at random. The Ethics Committees of the Korean Health and Genome Study and Ajou University School of Medicine approved the study protocol. Written informed consent was obtained from each participant.

\section{Data collection}

Demographic information collected at baseline included level of education, occupation, exercise frequency, and comorbidities using a standard questionnaire during a face-toface interview. Level of education was dichotomized into $\geq$ 12 years (finished high school, finished vocational school, some college, finished college, some graduate school and higher) or $<12$ years for the analysis. Occupation was classified into 14 standard categories according to the Korean National Statistical Office. Occupations including factory worker, laborer, and farmer were defined as manual work. 
The exercise category was self-reported and classified as never, once per week, two to three times per week, or daily. Height $(\mathrm{cm})$ and body weight $(\mathrm{kg})$ were measured to the nearest $0.1 \mathrm{~cm}$ and $0.1 \mathrm{~kg}$, respectively, with the subject wearing light clothing and barefooted for calculation of the body mass index (BMI). A BMI $\geq 30 \mathrm{~kg} / \mathrm{m}^{2}$ was defined as obesity.

The point prevalence of LBP was obtained using a questionnaire which asked, "Do you have LBP at the present time, that is, right now?", and a mannequin diagram was used to define the anatomical location of LBP. Additionally, a validated Korean version of the Oswestry disability index (ODI) was administered to measure disability resulting from $\mathrm{LBP}^{6}$.

\section{Radiographic assessment}

Lateral lumbar spine radiographs were taken according to a standard protocol with the film centered on the second lumbar vertebra. Each vertebral level from L1/2 to L4/5 was reviewed for radiographic features of degenerative changes. Semi-quantitative scores for the following features were given using a reference atlas ${ }^{7}$ : presence and severity of anterior osteophytes (grade $0=$ none; grade $1=$ barely visible; grade $2=$ definite; grade $3=$ large), endplate sclerosis (grade $0=$ none; grade $1=$ present), and joint-space narrowing (grade $0=$ none; grade $1=$ probable; grade $2=$ definite; grade $3=$ severe, bone to bone). Additionally, KL grading was used for each vertebral level (grade $0=$ normal disc with no osteophytes; grade $1=$ slight anterior wear and osteophyte formation; grade $2=$ definite anterior wear and mild disc-space narrowing with osteophyte formation; grade $3=$ moderate disc-space narrowing with osteophytes and endplate sclerosis; grade $4=$ large osteophytes, marked disc-space narrowing, and endplate sclerosis). Radiographs were read by a single academically based rheumatologist (Jung YO). Intra-observer reproducibility was assessed by reevaluating 50 films within 1 week of the first reading. The reproducibility of intra-reader assessments was high (for osteophyte grading, $\kappa=0.89-0.93$; for endplate sclerosis, $\kappa=0.71-$ 84; for joint-space narrowing, $\kappa=0.81-0.89$; and for KL grading, $\kappa=0.69-0.80$, for various vertebral levels). Any discrepancies between the two readings were adjudicated by consensus between the original reader and the second reader.

\section{Statistical analysis}

For comparisons of the normal subjects to those with LS, continuous variables were tested using Student's t-test and categorical variables using Pearson's chi-squared test. ORs and 95\% confidence intervals for LS risk factors were calculated using univariate logistic regression analysis. In our univariate analysis, age, gender, hypertension, DM, smoking, manual work and self-reported arthritis were significantly associated with the presence of LS. KL grade $\geq 3$ LS was additionally significantly associated with obesity and exercise. Stepwise logistic regression analysis with a backward method was performed by testing the deletion of each variable until no further improvement in the model was observed. The Goodness of fit for the logistic regression was tested with Hosmer-Lemeshow test. The $p$-values derived were 0.976 for KL grade $\geq 2$ LS and 0.922 for KL grade $\geq 3$ LS, which indicated that the model fits the data well. Age was categorized into $\leq 59,60-69$ and $\geq 70$, because this resulted in similar number of participants in each category and because we observed that the prevalence of LBP increased according to this categorization in our previous study ${ }^{22}$.

The association between individual radiographic features and LBP was determined according to gender and age. The grade of the most severely affected vertebral level within each individual was used for the analysis. The correlation between LS and the severity of LBP as measured with ODI was analyzed using linear association after adjusting for age and gender.

Statistical analyses were performed using the SPSS software (ver. 12.0; SPSS Inc., Chicago, IL, USA). A $p$-value $<0.05$ (two-tailed) was considered to indicate statistical significance.

\section{RESULTS}

Table 1 shows the baseline characteristics of the study participants. The mean age of the study subjects was 61.4 years and $58.1 \%$ were females. Table 2 shows the prevalence and grade of individual radiographic features of LS. Grade $\geq 2$ os- 
Table 1. Baseline characteristics of the subjects

\begin{tabular}{|c|c|c|c|c|}
\hline Variable & All $(n=1512)$ & Men $(n=633)$ & Women $(n=879)$ & $p$-value \\
\hline Age (years, mean $\pm S D$ ) & $61.4 \pm 8.6$ & $61.6 \pm 8.4$ & $61.2 \pm 8.7$ & 0.456 \\
\hline Body mass index $\left(\mathrm{kg} / \mathrm{m}^{2}\right.$, mean $\left.\pm \mathrm{SD}\right)$ & $24.06 \pm 3.15$ & $23.31 \pm 2.91$ & $24.61 \pm 3.20$ & $<0.001$ \\
\hline Education $\geq 12$ years & $361(23.9)$ & $236(37.3)$ & $125(14.2)$ & $<0.001$ \\
\hline Manual occupation & $410(27.1)$ & $97(15.3)$ & $313(35.6)$ & $<0.001$ \\
\hline Regular exercise & $488(32.3)$ & $211(33.3)$ & $277(31.6)$ & 0.483 \\
\hline Previous or current smoker & $257(17.0)$ & $242(38.2)$ & $15(1.7)$ & $<0.001$ \\
\hline Alcohol & $607(40.2)$ & $419(66.2)$ & $188(21.4)$ & $<0.001$ \\
\hline Married & $1283(84.9)$ & $600(94.8)$ & $683(77.7)$ & $<0.001$ \\
\hline Diabetes mellitus & $337(22.3)$ & $126(19.9)$ & $211(24.0)$ & 0.132 \\
\hline Hypertension & $293(19.4)$ & $96(15.2)$ & $197(22.4)$ & $<0.001$ \\
\hline Self-reported hand or knee arthritis & $684(46.2)$ & $234(37.6)$ & $450(52.5)$ & $<0.001$ \\
\hline Presence of LBP & $552(36.5)$ & $166(26.2)$ & $386(43.9)$ & $<0.001$ \\
\hline
\end{tabular}

Except where indicated otherwise, values are presented as number (\%). The body mass index is the weight in kilograms divided by the square of the height in meters. Manual occupation was defined as work demanding physical exertion (factory worker, laborer and farmer). Regular exercise was defined as selfreported exercise more than 3 times per week. $p$ value denotes the difference between men and women. SD : standard deviation, LBP : Iower back pain

Table 2. Prevalence of lumbar spondylosis

\begin{tabular}{|c|c|c|c|c|}
\hline & Men $(n=633)$ & Women $(n=879)$ & All $(n=1512)$ & $\operatorname{LBP}(n=552)$ \\
\hline \multicolumn{5}{|c|}{ Osteophytes (\%) } \\
\hline Grade 0 & $8(1.3)$ & $68(7.7)$ & $76(5.0)$ & $27(4.9)$ \\
\hline Grade 1 & $215(34.0)$ & $406(46.2)$ & $621(41.1)$ & $192(34.8)$ \\
\hline Grade 2 & $232(36.7)$ & $270(30.7)$ & $502(33.2)$ & $206(37.3)$ \\
\hline Grade 3 & $178(28.1)$ & $135(15.4)$ & $313(20.7)$ & $127(23.0)$ \\
\hline \multicolumn{5}{|c|}{ Joint space narrowing (\%) } \\
\hline Grade 0 & $290(45.8)$ & $402(45.7)$ & $692(45.8)$ & $220(39.9)$ \\
\hline Grade 1 & $246(38.9)$ & $335(38.1)$ & $581(38.4)$ & $210(38.0)$ \\
\hline Grade 2 & 78 (12.3) & $126(14.3)$ & $204(13.5)$ & $102(18.5)$ \\
\hline Grade 3 & $19(3.0)$ & $16(1.8)$ & $35(2.3)$ & $20(3.6)$ \\
\hline \multicolumn{5}{|c|}{ KL grade (\%) } \\
\hline Grade 0 & $9(1.4)$ & $67(7.6)$ & $76(5.0)$ & $26(4.7)$ \\
\hline Grade 1 & 99 (15.6) & $236(26.8)$ & $335(22.2)$ & $111(20.1)$ \\
\hline Grade 2 & $292(46.1)$ & $369(42.0)$ & $661(43.7)$ & $221(40.0)$ \\
\hline Grade 3 & $188(29.7)$ & $173(19.7)$ & $361(23.9)$ & $153(27.7)$ \\
\hline Grade 4 & $45(7.1)$ & $34(3.9)$ & $79(5.2)$ & $41(7.4)$ \\
\hline
\end{tabular}

Values are presented as number (\%). LBP : lower back pain, KL : Kellgren-Lawrence

teophytes and joint-space narrowing (JSN) were present in 53.9 and $15.8 \%$ of subjects, respectively. Seventy-three percent of subjects had KL grade $\geq 2$ degenerative changes $(83 \%$ among males, $65.6 \%$ among females). Male predominance in LS prevalence was more pronounced in advanced stage, with about twofold difference in KL grade 4 and osteophyte/JSN grade 3 prevalence between males and females. LBP was present in $36.5 \%$ of study subjects $(26.2 \%$ among males and 43.9\% among females). Demographic factors associated with the presence of LS are shown in Table 3. LS defined as KL grade $\geq 2$ was significantly associated with age, manual work, hypertension and self-reported history of hand or knee ar- 
Prevalence and Risk Factors for LS and Its Association with LBP among Rural Korean Residents | Lee SY, et al.

Table 3. Risk factors of lumbar spondylosis ( $K L$ grade $\geq 2$ in any level)

\begin{tabular}{|c|c|c|c|c|}
\hline & KL gra & & KL grad & \\
\hline & OR $(95 \% \mathrm{Cl})$ & $p$-value & OR $(95 \% \mathrm{CI})$ & $p$-value \\
\hline Age & & & & \\
\hline$\geq 50,<60$ (vs. $\geq 40,<50)$ & 1.165 (0.799-1.699) & 0.427 & $2.656(1.326-5.321)$ & 0.006 \\
\hline$\geq 60,<70($ vs. $\geq 40,<50)$ & $3.157(2.078-4.797)$ & 0.0001 & $5.761(2.902-11.434)$ & 0.0001 \\
\hline$\geq 70$ (vs. $\geq 40,<50$ ) & $4.806(2.888-7.996)$ & 0.0001 & $10.244(5.066-20.714)$ & 0.0001 \\
\hline Female & $0.369(0.279-0.487)$ & 0.0001 & $0.430(0.330-0.561)$ & 0.0001 \\
\hline Hypertension & $1.432(1.014-2.022)$ & 0.041 & $1.473(1.095-1.998)$ & 0.01 \\
\hline Self-reported hand, knee arthritis & $1.571(1.162-2.123)$ & 0.003 & $1.675(1.268-2.214)$ & 0.0001 \\
\hline Manual work & 1.477 (1.122-1.945) & 0.005 & - & 0.093 \\
\hline Obesity & - & NA & $2.217(1.195-4.113)$ & 0.012 \\
\hline Exercise & - & NA & $0.729(0.554-0.958)$ & 0.023 \\
\hline Smoking & - & 0.472 & - & 0.484 \\
\hline DM & - & 0.991 & - & 0.927 \\
\hline
\end{tabular}

Adjustments made for the factors significantly associated in the univariate analysis using stepwise logistic regression analysis with a backward method. NA denotes not applicable due to non-significance with univariate analysis. KL : Kellgren-Lawrence, OR : odds ratio, Cl : confidence interval, NA : not applicable, DM : diabetes mellitus

Table 4. Association between individual radiographic features of lumbar spondylosis and LBP according to age and gender

\begin{tabular}{|c|c|c|c|c|c|}
\hline \multirow{2}{*}{ Age (years) } & \multirow{2}{*}{ n (\%) } & \multirow{2}{*}{ LBP (\%) } & \multicolumn{3}{|c|}{ Odd ratio for LBP $(95 \% \mathrm{Cl})$} \\
\hline & & & Osteophyte $\geq 2$ & JSN $\geq 2$ & $K L$ grade $\geq 2$ \\
\hline \multicolumn{6}{|l|}{ All, $n=1512$} \\
\hline$\leq 59$ & $636(42.1)$ & $201(31.6)$ & 1 & 1 & 1 \\
\hline $60-69$ & $528(34.9)$ & $196(37.1)$ & $1.14(0.88-1.47)$ & $1.17(0.91-1.51)$ & $1.19(0.92-1.54)$ \\
\hline$\geq 70$ & $348(23.0)$ & $155(44.5)$ & $1.57(1.17-2.10)$ & $1.65(1.24-2.19)$ & $1.70(1.27-2.26)$ \\
\hline \multicolumn{6}{|l|}{ Men, $n=633$} \\
\hline$\leq 59$ & $267(42.2)$ & $63(23.6)$ & 1 & 1 & 1 \\
\hline $60-69$ & $214(33.8)$ & $52(24.3)$ & $0.93(0.60-1.44)$ & $0.88(0.57-1.36)$ & $1.00(0.65-1.54)$ \\
\hline$\geq 70$ & $152(24.0)$ & $51(33.6)$ & $1.45(0.91-2.31)$ & $1.45(0.92-2.30)$ & $1.59(1.00-2.52)$ \\
\hline \multicolumn{6}{|c|}{ Women, $n=879$} \\
\hline$\leq 59$ & $369(42.0)$ & $138(37.4)$ & 1 & 1 & 1 \\
\hline $60-69$ & $314(35.7)$ & $144(45.9)$ & $1.26(0.92-1.73)$ & 1.35 (0.99-1.85) & $1.30(0.95-1.79)$ \\
\hline$\geq 70$ & $196(22.3)$ & $104(53.1)$ & $1.61(1.11-2.35)$ & $1.81(1.25-2.61)$ & $1.75(1.21-2.53)$ \\
\hline
\end{tabular}

Adjustments made for body mass index. LBP : lower back pain, Cl : confidence interval, JSN : joint-space narrowing, KL : Kellgren-Lawrence

thritis, and negatively associated with female gender after adjustment. On the other hand, more advanced LS, defined as KL grade $\geq 3$, was significantly associated with age, hypertension, obesity, and self-reported history of hand or knee arthritis, and negatively associated with female gender and exercise. Next, we evaluated the relationship between individual radiographic features of LS and the presence of LBP according to age and gender (Table 4). The presence of grade $\geq 2$ osteophytes or JSN and KL grade $\geq 2$ was associated with LBP in subjects over 70 years old. However, this association was present mostly among females. Among males, LBP was significantly associated only with $\mathrm{KL}$ grade $\geq 2$. Lastly, we observed an association between LS and disability caused by LBP as measured with ODI (Fig. 1). After adjusting for age and gender, joint-space narrowing, osteophytes, and KL grade were all significantly correlated with ODI. 


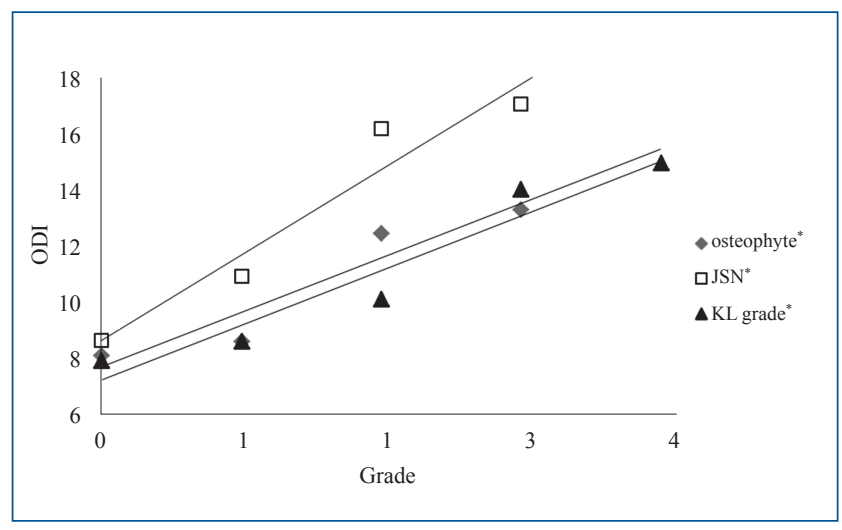

Fig. 1. Linear by linear association between individual radiographic features of lumbar spondylosis and ODI. The graph shows significantly positive linear association $\left({ }^{*} p<0.0001\right)$ after adjustment for age and gender. ODI : Oswestry disability index, JSN : joint-space narrowing, KL : Kellgren-Lawrence.

\section{DISCUSSION}

In this study of middle-to-old age rural Korean residents, the prevalence of LS defined as at least one intervertebral level of KL grade $\geq 2$ was $73 \%$. Signs of advanced spinal degeneration, such as grade 3 osteophytes and intervertebral discspace narrowing, were more frequent among males, although the prevalence of LBP was higher among females. The association between LS and LBP was observed mostly among females. LS was significantly correlated with the severity of LBP measured with ODI after adjusting for confounders.

Compared to a previous Japanese report from the ROAD cohort $^{14)}$, our study subjects were younger (mean age 69.671.0 vs. 61 years); however, the prevalence of LS was similar. Our study subjects only included rural community residents, and inclusion of urban residents may have resulted in an even higher prevalence of LS, as, in the ROAD study, urban residents had a higher prevalence compared to mountainous or seacoast residents.

In line with the Japanese report, males had a higher prevalence of LS defined as KL grade $\geq 2$. Unlike OA in peripheral joints, such as the hand and knee, degenerative spinal changes were reported to be more frequent in males than females ${ }^{14,17)}$. The gender difference was more conspicuous in cases of higher grade LS in our study. It is not known why the peripheral joint and spine are differentially affected by OA among males and females, although genetic and hormonal factors and their inter-relationship are speculated. It is also possible that compared to peripheral joints, the spine is more affected by strenuous occupations, such as outdoor work. Studies examining individual radiographic features of LS reported that while osteophytes were more frequent in males, the frequency of disc-space narrowing was either increased or similar in females ${ }^{3,9)}$. Discrepancies in individual radiographic features of LS among different studies may result from methodological or ethnic differences. In a study that compared the prevalence of LS between the Japanese and British populations, Japanese females tended to have a lower prevalence of joint-space narrowing compared to Japanese males $^{17)}$. On the other hand, a European study of 359 elderly subjects that used magnetic resonance imaging (MRI) to examine disc degeneration in the lumbar spine found that female subjects had more severe disc degeneration than male subjects ${ }^{13}$. The discrepancy may stem from the MRI grading system for disc degeneration based on signal intensity, disc structure, and distinction between the nucleus pulposus and annulus fibrosus, which are not evaluated by simple radiograph. It would be interesting to investigate whether an ethnic difference exists in the susceptibility to disc degeneration according to gender, as evaluated by MRI.

The risk factors of LS identified in this study are consistent with other studies that have included age and degree of manual work ${ }^{5)}$. An MRI study from Japan revealed that in addition to aging and occupational lifting, high BMI and high low-density lipoprotein cholesterol were associated with disc degeneration, suggesting that cardiovascular risk factors play a role in $\mathrm{LS}^{4)}$. In our study, hypertension and obesity were associated with more advanced LS, defined as KL grade $\geq 3$. Although heavy physical loading was traditionally suspected as a major risk factor for LS, recent studies suggest that occupational loading may play a relatively minor role. A retrospective twin study investigating the effects of occupational and leisure time physical loading on disc degeneration showed that the mean job code explained only $7 \%$ of the variability in disc degeneration scores1). The addition of age accounted for $16 \%$, and familial aggregation im- 
proved the model such that $77 \%$ of the variability was explained, indicating that heredity has a dominant role in disc degeneration.

Although the causal relationship between LBP and LS has been debated, the lumbar spine is routinely evaluated radiographically in patients with LBP. In an earlier systematic review of observational studies, LS was found to be associated with nonspecific LBP; however, methodological flaws concerning control of confounding factors and the blinded assessment of LBP status and of radiographs were present ${ }^{12}$. More recent reports examining individual radiographic features of LS consistently showed an association between discspace narrowing and osteophytes with LBP after adjustment for confounders ${ }^{3,9)}$. It is of note that LS was only associated with LBP in females older than 70 years in our study. This result contradicts those of de Schepper et al. ${ }^{3)}$, which showed a stronger association between LBP and disc-space narrowing in males compared with females. On the other hand, a Japanese study showed that symptomatic LBP was associated with KL grade $\geq 3$ spondylosis in females but not in males ${ }^{8}$. In agreement with other studies, LBP was considerably more prevalent among females in our study, and it is possible that LBP in females arises more often from problems other than disc-space narrowing, such as ligamentous strain or muscle weakness. This explanation is not exclusive of other hypotheses on gender difference, which state that LS is more prone to induce LBP in females due to decreased muscle strength which cannot compensate for spinal instability arising from degenerative changes ${ }^{8}$. In another study, anxiety was linked to self-reported and induced LBP in males but not in females, which may explain the lower association between LS and LBP in males ${ }^{10)}$.

Lastly, we observed that LS was significantly associated with the severity of LBP as assessed with ODI after adjusting for age and sex. This finding further suggests that LS is a significant factor determining the presence and severity of LBP.

Our study has strengths and limitations. We used individual radiographic features to detect LS and analyzed its association with LBP in a large sample of Korean residents. We included elderly subjects, an under-represented group in back pain research. By using a validated Korean version of ODI, we verified the association between LS and the severity of LBP for the first time, to our knowledge. Limitations include the inclusion of only rural residents, which limit the generalizability of the findings. Due to budget restraints, we were unable to obtain lumbar radiographs of all of our study subjects and had to randomly select those to be radiographed, which could have created selection bias. Although the LBP rate was not different between those who were radiographed and those who were not, included subjects were significantly older compared to non-included subjects, which may have led to over-estimation of the LS prevalence. We were unable to evaluate facet joints in the lumbar spine radiographs due to technological limitations, and a recent report showing degeneration beginning in the facet joints in $22 \%$ of study subjects raises the concern that we may have missed a significant portion of atypical-pattern LS subjects ${ }^{11}$.

\section{CONCLUSION}

Our study among rural Korean residents showed that the prevalence of LS was 73\%, and was and significantly correlated with the severity of back pain measured with ODI. The association between LS and LBP was observed only among females, which may reflect the gender difference in the pathogenesis of pain in musculoskeletal condition. This is an important area to pursue in the future research. In addition, the search of preventive measures for LS and LBP including better work ergonomics is urgently needed for our ever-aging population.

\section{- Acknowledgments}

This study was supported by a grant from the National Genome Research Institute, the Korean Center for Disease Control and Prevention (contract \#2001 2003-348-6111-221, 2004-347-6111-213 and 2005-347-2400-2440-215) and a grant from the Korea Health Technology R \& D project, Ministry of Health and Welfare (A120960). 


\section{- Supplementary Materials}

The online-only Data Supplement is available with this article at https://doi.org/10.3340/jkns.2016.0505.007.

\section{References}

1. Battié MC, Videman T, Gibbons LE, Fisher LD, Manninen H, Gill K : 1995 Volvo Award in clinical sciences. Determinants of lumbar disc degeneration. A study relating lifetime exposures and magnetic resonance imaging findings in identical twins. Spine (Phila Pa 1976) 20 : 2601-2612, 1998

2. Cho NH, Jung YO, Lim SH, Chung CK, Kim HA : The prevalence and risk factors of low back pain in rural community residents of Korea. Spine (Phila Pa 1976) 37 : 2001-2010, 2012

3. de Schepper El, Damen J, van Meurs JB, Ginai AZ, Popham M, Hofman A, et al. : The association between lumbar disc degeneration and low back pain: the influence of age, gender, and individual radiographic features. Spine (Phila Pa 1976) 35 : 531-536, 2010

4. Hangai M, Kaneoka K, Kuno S, Hinotsu S, Sakane M, Mamizuka N, et al. : Factors associated with lumbar intervertebral disc degeneration in the elderly. Spine J 8 : 732-740, 2008

5. Jhun HJ, Park JY : Estimated number of korean adults with back pain and population-based associated factors of back pain : data from the fourth Korea national health and nutrition examination survey. J Korean Neurosurg Soc 46 : 443-450, 2009

6. Jeon CH, Kim DJ, Kim SK, Kim DJ, Lee HM, Park HJ : Validation in the cross-cultural adaptation of the Korean version of the Oswestry Disability Index. J Korean Med Sci 21 : 1092-1097, 2006

7. Lane NE, Nevitt MC, Genant HK, Hochberg MC : Reliability of new indices of radiographic osteoarthritis of the hand and hip and lumbar disc degeneration. J Rheumatol 20 : 1911-1918, 1993

8. Muraki S, Oka H, Akune T, Mabuchi A, En-Yo Y, Yoshida M, et. al. : Prevalence of radiographic lumbar spondylosis and its association with low back pain in elderly subjects of population-based cohorts: the ROAD study. Ann Rheum Dis 68 : 1401-1406, 2009

9. Pye SR, Reid DM, Smith R, Adams JE, Nelson K, Silman AJ, et al. : Radiographic features of lumbar disc degeneration and self-reported back pain. J Rheumatol 31 : 753-758, 2004

10. Robinson ME, Dannecker EA, George SZ, Otis J, Atchison JW, Fillingim $\mathrm{RB}$ : Sex differences in the associations among psychological factors and pain report: a novel psychophysical study of patients with chronic low back pain. J Pain 6 : 463-470, 2005

11. Suri P, Miyakoshi A, Hunter DJ, Jarvik JG, Rainville J, Guermazi A, et al. : Does lumbar spinal degeneration begin with the anterior structures? A study of the observed epidemiology in a community-based population.

BMC Musculoskelet Disord 12 : 202, 2011

12. van Tulder MW, Assendelft WJ, Koes BW, Bouter LM : Spinal radiographic findings and nonspecific low back pain. A systematic review of observational studies. Spine (Phila Pa 1976) 22 : 427-434, 1997

13. Wang YX, Griffith JF, Ma HT, Kwok AW, Leung JC, Yeung DK, et al. : Relationship between gender, bone mineral density, and disc degeneration in the lumbar spine: a study in elderly subjects using an eight-level MRIbased disc degeneration grading system. Osteoporos Int 22 : 91-96, 2011

14. Yoshida S, Aoyagi K, Felson DT, Aliabadi P, Shindo H, Takemoto T : Comparison of the prevalence of radiographic osteoarthritis of the knee and hand between Japan and the United States. J Rheumatol 29 : 14541458,2002

15. Yoshimura N, Dennison E, Wilman C, Hashimoto T, Cooper C : Epidemiology of chronic disc degeneration and osteoarthritis of the lumbar spine in Britain and Japan: a comparative study. J Rheumatol 27 : 429-433, 2000

16. Yoshimura N, Muraki S, Oka H, Mabuchi A, En-Yo Y, Yoshida M, et al. : Prevalence of knee osteoarthritis, lumbar spondylosis, and osteoporosis in Japanese men and women: the research on osteoarthritis/osteoporosis against disability study. J Bone Miner Metab 27 : 620-628, 2009

17. Zhang Y, Xu L, Nevitt MC, Aliabadi P, Yu W, Qin M, et al. : Comparison of the prevalence of knee osteoarthritis between the elderly Chinese population in Beijing and whites in the United States: The Beijing Osteoarthritis Study. Arthritis Rheum 44 : 2065-2071, 2001 